\title{
A Brief Overview and Future Perspective of Unmanned Aerial Systems for In-Service Structural Health Monitoring
}

\author{
Meisam Gordan', Zubaidah Ismail,",, Khaled Ghaedi',2, Zainah Ibrahim', Huzaifa Hashim1, Haider \\ Hamad Ghayeb', Marieh Talebkhah ${ }^{3}$ \\ ${ }^{1}$ Department of Civil Engineering, University of Malaya, 50603, Kuala Lumpur, Malaysia. \\ ${ }^{2}$ Center of Research \& Development, PASOFAL Engineering, 52200, Kuala Lumpur, Malaysia. \\ ${ }^{3}$ Department of Computer and Communication Systems Engineering, Universiti Putra Malaysia, 43400, Malaysia.
}

How to cite this paper: Meisam Gordan, Zubaidah Ismail, Khaled Ghaedi, Zainah Ibrahim, Huzaifa Hashim, Haider Hamad Ghayeb, Marieh Talebkhah. (2021) A Brief Overview and Future Perspective of Unmanned Aerial Systems for In-Service Structural Health Monitoring. Engineering Advances, 1(1), 9-15.

DOI: 10.26855/ea.2021.06.002

Received: December 24, 2020

Accepted: January 26, 2021

Published: February 24, 2021

"Corresponding author: Zubaidah Ismail, Department of Civil Engineering, University of Malaya, 50603, Kuala Lumpur, Malaysia.

Email: zu ismail@um.edu.my

\begin{abstract}
Remote sensing with Unmanned Aerial Systems (UASs) is a game-changer in various fields such as environmental monitoring, surveillance, aerial photography, digital communications, search and rescue operations and military. This paper presents one of the most economical and yet the most effective approaches used in Structural Health Monitoring (SHM). Herein, a review of the recent advances, applications and future perspective of UASs, i.e. drones in SHM is discussed. Drones have become popular in several developed countries in recent years. However, the use of drones is still in the infancy stage of development in developing countries. The development of drones in the last decade has marked a new era in remote sensing, providing data of unprecedented spatial, spectral, and temporal resolution. This is due to the fact that UASs are low cost aerial robots, that require little preparation and infrastructure and can be equipped with any number of sensors or cameras making them ideal for monitoring the environment. To this end, drones offer an opportunity to infrastructures the existing gap between field observations and remote sensing by providing high spatial detail over relatively large areas in a cost-effective way.
\end{abstract}

\section{Keywords}

Structural Health Monitoring, Unmanned Aerial Systems, Internet of Drone, Remote sensing

\section{Introduction}

The potential for Unmanned Aerial Systems (UAS)-based generation of high-quality images of structural details to detect structural damage and support condition assessment of civil structures is very high, particularly in difficult-to-access areas. For example, drone-based visual inspection is used for monitoring oil and gas platforms [1], construction sites [2], infrastructures like bridges [3] and buildings [4], pipelines [5], cracks in concrete civil infrastructures [6], etc. Integration of Unmanned Aerial Vehicle (UAV) is also being exploited for industrial inspection tasks [7].

Drones are becoming increasingly popular for commercial and non-commercial uses, especially in the fields of environment, surveillance, aerial photography, digital communications, search and rescue operations and military. Drones are in fact low cost aerial robots, that require little preparation and infrastructure and can be equipped with any number of sensors or cameras making them ideal for monitoring the environment. Environmental monitoring plays a major role in analyzing climate and management impacts on natural, agricultural systems, assessing, forecasting and 
even preventing natural disasters and enhancing hydrological cycle. Monitoring and data collection systems are based upon a combination of ground-based measurements and remote sensing sensors observations. These data however have spatiotemporal constraints. Drones offer an opportunity to infrastructures, e.g. bridge the existing gap between field observations and remote sensing by providing high spatial detail over relatively large areas in a cost-effective way. Drones have become popular in several developed countries in recent years. However, the use of drones is still in the infancy stage of development in developing countries such as Malaysia [8].

The followings are covered in the rest of the present study: a brief overview of remote sensing is presented in Section 2, while the UASs are detailed in Section 3. Section 4 is addressed the future perspectives and works. Last but not least, the conclusion is drawn in Section 5.

\section{Remote Sensing Technology}

Remote sensing is defined as the analysis of object properties, area or phenomenon on the earth's surface through data acquired from a device that is not in contact with the object, area, or phenomenon under investigation, i.e. terrestrial aircraft and satellites in order to obtain information about the asset. This may be associated with the fact that it is usually very difficult to obtain an immediate assessment of large-scale natural disasters using ground survey alone. Consequently, remote sensing by airborne or spaceborne sensors is often applied to monitor near-real-time damage for large-scale events. The current generation of remote sensing satellites is producing data with great potential for use in scientific and technological investigations in very large and ever increasing quantities. Remote sensing of the Earth has many applications such as environmental assessment and monitoring and agriculture. Many remote sensing systems have been developed to offer a wide range of spatial, spectral, and temporal parameters. For the past fifty years, several Commercial Remote Sensing (CRS) and Spatial Information (SI) technologies for wide-bandwidth spectral information sensing and imaging have been developed integrally with satellite/airborne/ground-based surveillance platforms [9-11].

Most of the data used in the representative research are in high resolution and can be classified into four categories: (1) optical; (2) Synthetic Aperture Radar (SAR); (3) Light Detection And Ranging (LiDAR); and (4) ancillary data. The ancillary data include maps produced from GIS data acquired by remote sensing and in situ damage assessment [12]. High-resolution multispectral optical imagery is easy to interpret because it provides a close representation of what the human eye sees. Conversely, micro-wave imaging radar such as SAR provides operational advantages over optical sensors for rapid disaster assessment because of its day/night and all-weather acquisition capability with wide coherent imaging coverage $[10,13]$. Figure 1 presents several remote sensing examples.
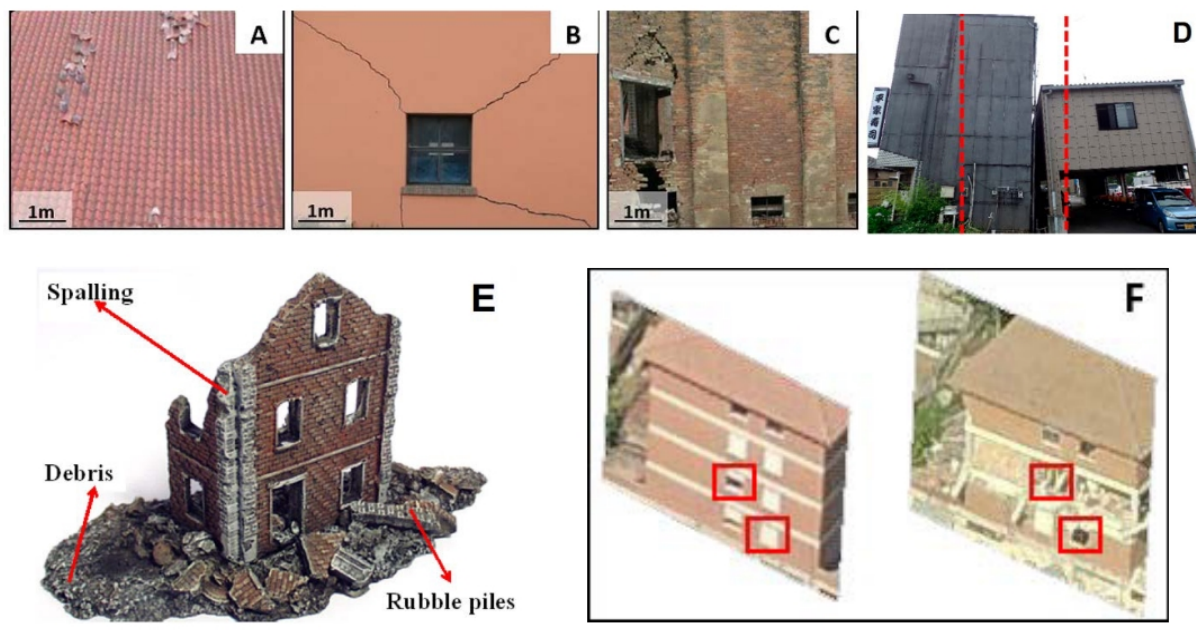

Figure 1. Examples of remote sensing (A) Roof with dislocated tiles, (B) cracks in concrete facade, (C) cracks and hole in brick facade, (D) inclined building, E) debris, rubble piles, spalling, and (F) facade windows symmetry. [13]

Data mining is one of the emerging data extraction techniques [14]. The application of data mining in remote sensing has been reported by [11]. In this study, the authors established that data mining techniques, e.g. Artificial Neural Network (ANN), decision trees, and clustering found patterns based on each algorithm's computational ability and they were not guided by user interaction. Therefore, they achieved a better performance. In addition, it was concluded that data mining techniques have the potential to improve spectral classification in remote sensing. In the same line, according to [15], ANN has the highest application rate for different functions. The reason for this comes from the fact that the ANN has proven its high capacities to work out in various purposes through pattern recognition [16-22]. Besides, various metaheuristic algorithms, e.g. Particle Swarm Optimization (PSO) [23], Genetic Algorithm (GA) [24], and Imperialist Competitive Algorithm (ICA) [25] have been developed to solve a variety of engineering optimization problems in SHM [26]. 


\section{Unmanned Aerial Systems (UASs)}

Remote sensing with Unmanned Aerial Systems (UASs) is a game-changer in many domains. The development and steep rise of drones in the last decade has marked a new era in remote sensing, providing data of unprecedented spatial, spectral, and temporal resolution [27]. Drones can be classified into multiple categories depending on their functions, size, weight, number of propellers, aerodynamic flight principles, range, equipment, cameras used and so on. Considering the operations accompanied to the urban planning sector, the prominent types of drones are rotary-wing and fixed-wing drones, where the classification is based on the differences in their aerodynamic flight principles. The rotary-wing motors are more common, as they offer high accessibility, ease of use, good camera control, ability to function in confined spaces and supports hover and Vertical Take-Off and Landing (VTOL); cost-wise also these are very affordable options. Depending on the rotor configurations, the rotary-wing drones can be further classified into tricopters, quadcopters [Figure 2(a)], hexacopters [Figure 2(b)], Figure 3, etc. However, all these drones have limited flight time (of not more than 30 minutes); and small payload capacity (mostly below 8kg). Below show a few examples of drones.

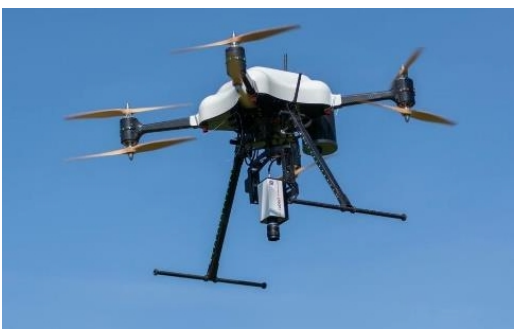

(a)

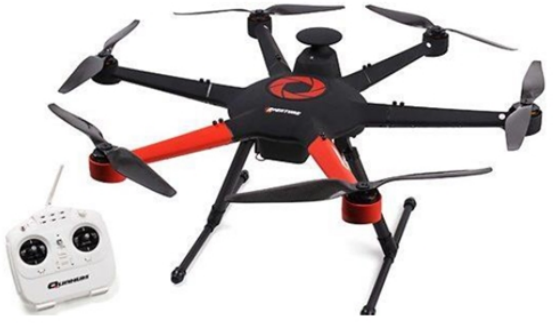

(b)

Figure 2. Drones (part 1): (a) Quadcopter Drone and (b) Hexcopter Drone [8].
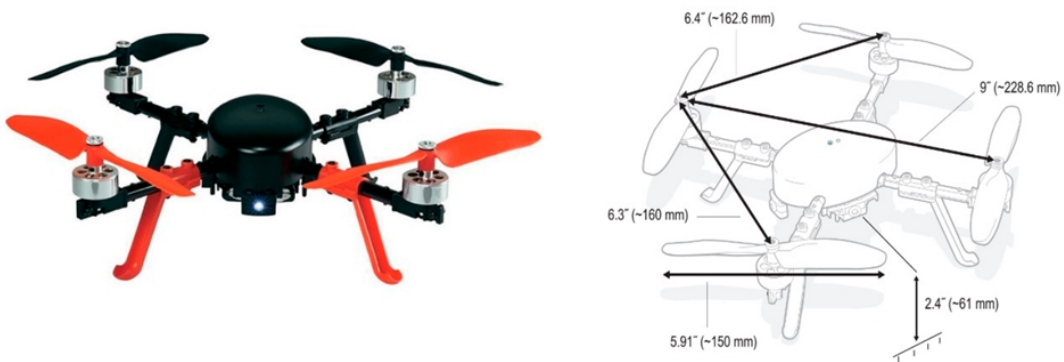

(a)

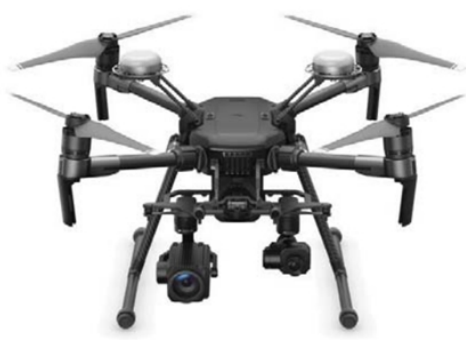

(b)

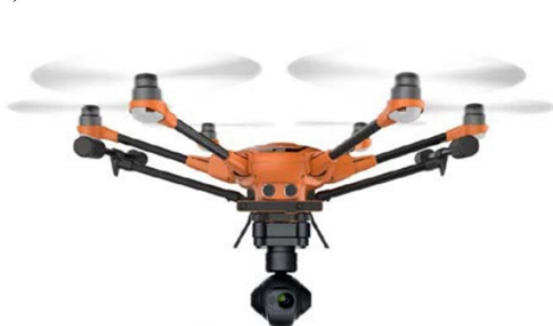

(c)

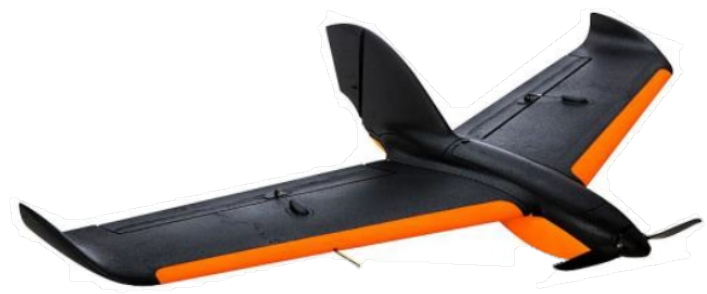

(d)

Figure 3. Drones (part 2): (a) Micro-UAV quadrotor, model "RC EYE One Xtreme" [7], (b). DJI Matrice 210 RTK with dual gimbal [28], (c) Unmanned aerial vehicle, model Yuneec H520 [29], and (d) Fixed-wing Drone [8]. 
According to [30], the key components of any drone are sensors. Only sensor systems with acceptable performance produce commercial drones the flight characteristics they need. Various tasks require various sensors or rather different sensor combinations. Some of the sensor types applied in drones include the following:

(1) LiDAR that can be used for collision avoidance and navigation, and also for a three-dimensional scanner,

(2) Cameras that can be used for observation, collision avoidance, data acquisition and navigation,

(3) Pressure gauges that can be used for navigation and data collection, and

(4) Global Positioning System (GPS) that can be used for position determination.

In the past several years, the medium spatial resolution SAR intensity images, such as ERS SAR, Envisat ASAR and Radarsat-1 SAR, have been used to study building damages in urban areas due to natural disasters, i.e. earthquake. Visual interpretation and change detection between remote sensing images acquired pre- and post-earthquake are important methods for damage assessment [31]. The suitable data source lies on the size of damaged target to be detected. Bad weather conditions usually limit the acquisition of optical remote sensing images, while all day and all weather SAR shows the ability of providing timely remote sensing data for emergency response and rescue works after earthquake. Because SAR is sensitive to the surface changes caused by earthquake, the modified electromagnetic behavior by geological disasters and the collapse of buildings can be recorded in SAR images as backscattering intensity changes [32].

A Laser Speckle Imaging System (LSIS) has been developed by [33] to achieve non-contact, non-destructive and remote strain sensing. This study demonstrated the potential application of LSIS as an effective NDT technique for full-field, non-contact and remote sensing of anisotropic deformation around fastener holes. Figure 4 presents the experimental set-up and instrumentation for LSIS.

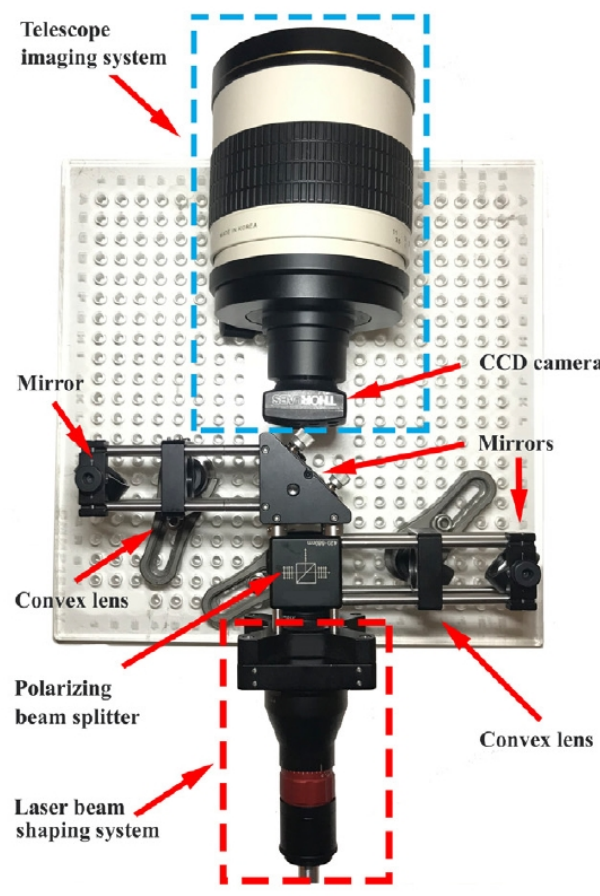

(a)

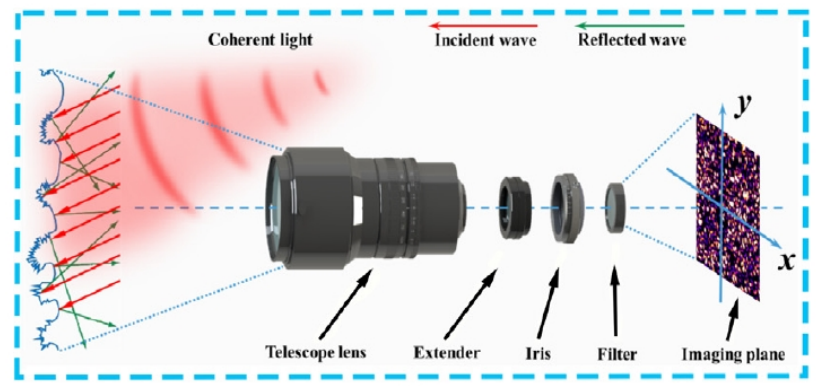

(b)

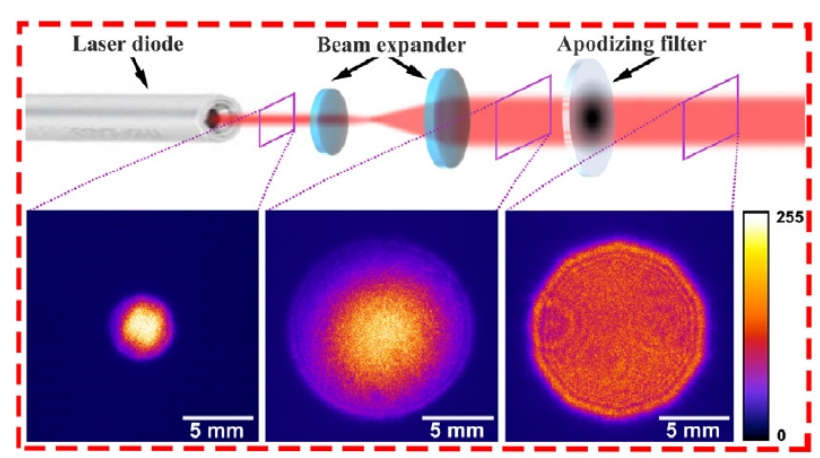

(c)

Figure 4. (a) Development of optical sensor for non-contact, non-destructive and remote strain sensing, (b) imaging system and principle to capture subjective laser speckle patterns, and (c) laser beam shaping system to obtain satisfactory beam size and even illumination [31].

A general physical structure of a drone was presented by [30], as shown in Figure 5. Each drone has computing power, recorder, energy supply, communication module, sensors and actuators. The drone also has a defined fly zone in which it can fly and send the required information to the control room. The internal user seating in the control room controls the drone remotely. The inbuilt sensors in the drone send the physical phenomena, such as temperature and concentration of hazardous gases, and the inbuilt camera in the drone takes the photographs or capture videos of the target and sends all information to the drone box via some wireless communication technology, such as WiFi. Each drone box is further connected to the server, which is then connected to the control room. 


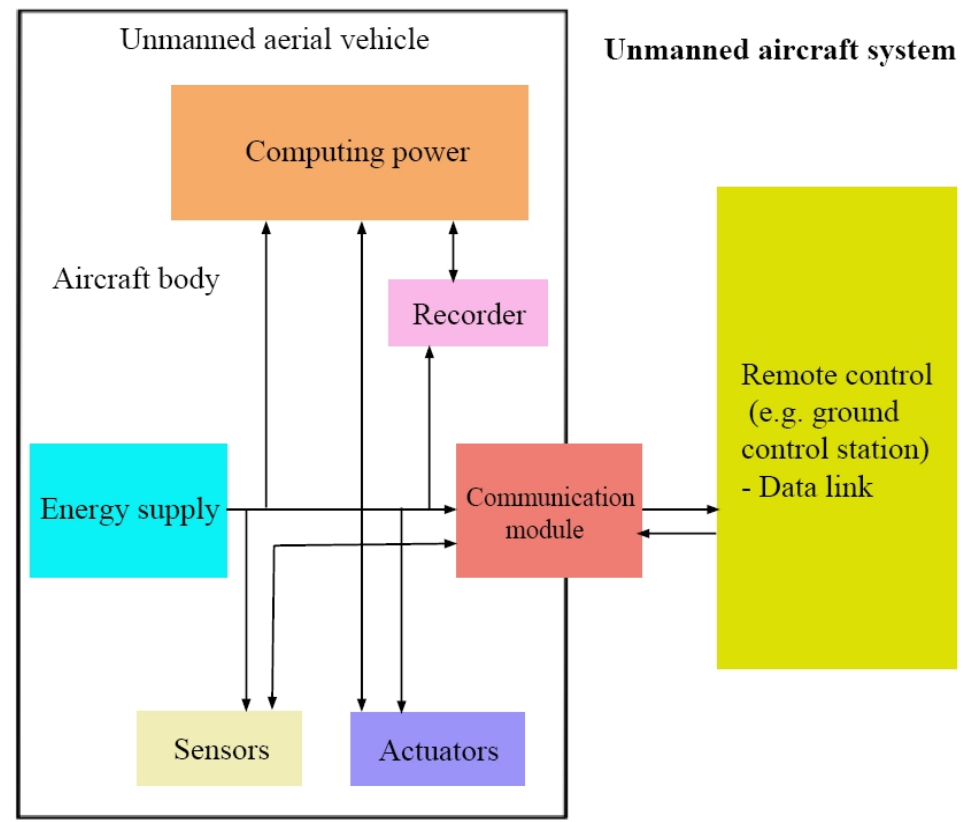

Figure 5. A general physical structure of a drone [30].

Several UAV-based systems were recommended by [29] to assist practically in routine bridge inspection work due to their stability, relatively superior performance as compared to other aircraft in the prosumer class, and for their ability to adapt software for automatic flight waypoints and 3D mapping of objects (see Figure 6).

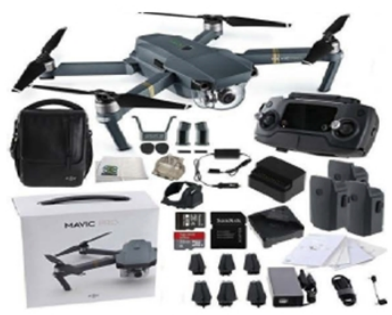

DJI Pro

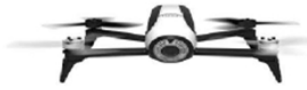

Parrot Bebop 2

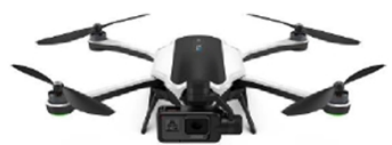

GoPro Karma

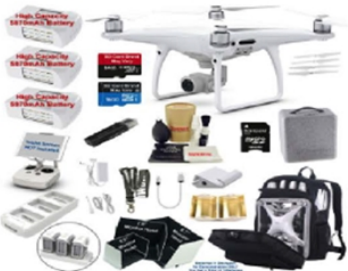

DJI Phantom 4 Pro

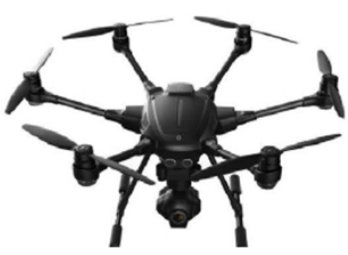

Yuneec Typhoon $\mathrm{H}$

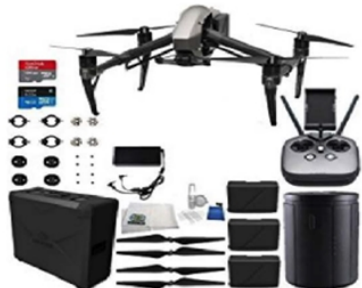

DJI Inspire 2

Figure 6. Introduced drones/UAVs by [29].

\section{Future Perspective}

The drone should consolidate rigid design, modern aeronautic technology as well as easy maintainability into a versatile multi-rotor platform, and durable enough to survive most crashes. The weight, model as well as energy source of a drone are typically the major components that impact its several factors, such as maximum altitude, flight range, flight duration along with maximum payload. The sensors are treated as a crucial category of payloads. Majority of drones are now equipped with cameras. While buying a drone, the cameras and microphones are the most frequently used payloads for drones and these commonly come as standard. Cameras can be also infrared and thus, such types of cameras may enable night vision as well as heat sensing too. Other sensors used in the drones include biological sensors 
that can detect microorganisms and meteorological sensors that can also measure various parameters, such as temperature, wind and humidity. In addition, cameras can be utilized for payloads in order to apply for the prevention, criminal investigation, criminal prosecution, and also sentencing of criminal behavior. Moreover, Internet of Things (IoT), Mobile Edge Computing (MEC), and fog computing are changing the physical world with traditional societies and industries to one huge database system which can support real-time applications [34]. In the same line, most applications in the Internet of Drone (IoD) environment assume drones to be flying camera surveillance [30]. The most important future challenges are to answer these questions, as follows:

(1) What technological solutions are able to simulate communications between drones?

(2) How to design a drone with full functionality for difficult-to-access areas?

(3) How to achieve the optimal characteristics of the drone, inlcuding its computing power, energy supply, communication module, sensors, actuators, cameras used, flight principles, and flight range?

\section{Conclusion}

This paper investigated the role of remote sensing and unmanned aerial vehicles in SHM. Based on previously mentioned explanations, an UAS device needs to be proposed to ensure the sustainability and resiliency of the implementation. In this regards, a physical structure of drone should be designed using various factors such as functionality for difficult-to-access areas, computing power, recorder, energy supply (battery and payload capacity), communication module, inbuilt sensors, actuators, size, weight, number of propellers, aerodynamic flight principles, range, equipment, cameras used, altitude, endurance, etc. This vehicle (drone) should consolidate rigid design, modern aeronautic technology as well as easy maintainability into a versatile multi-rotor platform, durable enough to survive most crashes. A low-level built-in flight control system can balance out small undesired changes to the flight altitude, and allows one to select three flight modes: beginner, sport and expert. Therefore, it is required to select the flying mode of the UAV for experimental data sessions.

\section{Acknowledgements}

This research was funded by the University of Malaya (UM) and the Ministry of Higher Education (MOHE), Malaysia [grant number: IIRG007A]. Accordingly, the authors would like to express their sincere thanks to Advance Shock and Vibration Research Group (ASVR), UM and MOHE.

\section{References}

[1] C. A. Marinho, C. De Souza, T. Motomura, A. G. da S. Silva, (2012). In-service flares inspection by unmanned aerial vehicles (UAVs), in: 18th World Conf. Nondestruct. Test., Durban, South Africa, 2012: pp. 16-20.

[2] R. R. S. de Melo, D. B. Costa, J. S. Álvares, J. Irizarry. (2017). Applicability of unmanned aerial system (UAS) for safety inspection on construction sites, Saf. Sci. 98(2017), 174-185. doi:10.1016/j.ssci.2017.06.008.

[3] N. Metni, T. Hamel. (2007). A UAV for bridge inspection: Visual servoing control law with orientation limits, Autom. Constr., 17(2007), 3-10. doi:10.1016/j.autcon.2006.12.010.

[4] C. Eschmann, C. M. Kuo, C. H. Kuo, C. Boller. (2012). Unmanned aircraft systems for remote building inspection and monitoring, Saarbrücken, Germany, 2012.

[5] S. Sadovnychiy. (2004). Count buffon on cultural changes of the physical environment, in: 8th WSEAS Int. Conf. Syst., Athens, Greece, 2004. doi:10.1111/j.1467-8306.1960.tb00325.x.

[6] H. Kim, S. H. Sim, S. Cho. (2015). Unmanned aerial vehicle (UAV)-powered concrete crack detection based on digital image processing, in: 6th Int. Conf. Adv. Exp. Struct. Eng., Urbana-Champaign, United States, 2015.

[7] U. Papa, S. Ponte. (2018). Preliminary design of an unmanned aircraft system for aircraft general visual inspection, Electron. 7 (2018). doi: 10.3390/electronics7120435.

[8] A. A. Ab Rahman, W. S. Wan Mohd Jaafar, K. N. Abdul Maulud, N. M. Noor, M. Mohan, A. Cardil, C. A. Silva, N. N. Che'Ya, N. I. Naba. (2019). Applications of Drones in Emerging Economies: A case study of Malaysia, in: 6th Int. Conf. Sp. Sci. Commun. Iconsp., Johor Bahru, Malaysia, 2019. doi:10.1109/IconSpace.2019.8905962.

[9] S. Chen, Y. Tong. (2012). Integrated Remote Sensing and Visualization: Phase Two, Web-GIS Based Bridg. Inf. Database. 4 (2012). https://ntl.bts.gov/lib/54000/54800/54876/PhaseII_USDOT_UNCC_Vol4-FinalRpt.pdf.

[10] S. Park, Y. Yamaguchi, D. Kim. (2013). Remote Sensing of Environment Polarimetric SAR remote sensing of the 2011 Tohoku earthquake using ALOS/PALSAR, Remote Sens. Environ. 132 (2013) 212-220. doi:10.1016/j.rse.2013.01.018.

[11] R. Kumar Sing. (2005). Pattern Recognition in Remote-Sensing Imagery using Data Mining and Statistical Techniques, PhD Thesis, Purdue University, 2005.

[12] L. Dong, J. Shan. (2013). A comprehensive review of earthquake-induced building damage detection with remote sensing 
techniques, ISPRS J. Photogramm. Remote Sens. 84 (2013), 85-99. doi:10.1016/j.isprsjprs.2013.06.011.

[13] S. Ghaffarian, N. Kerle, T. Filatova. (2018). Remote sensing-based proxies for urban disaster risk management and resilience: A review, Remote Sens. 10(2018). doi: 10.3390/rs10111760.

[14] M. Gordan, Z. Ismail, H. A. Razak, Z. Ibrahim. (2017). Vibration-Based Structural Damage Identification Using Data Mining, in: 24th Int. Congr. Sound Vib. London, 2017.

[15] M. Gordan, H. A. Razak, Z. Ismail, K. Ghaedi. (2017). Recent developments in damage identification of structures using data mining, Lat. Am. J. Solids Struct. 14(2017), 2373-2401. doi:10.1590/1679-78254378.

[16] M. Gordan, H. A. Razak, Z. Ismail, K. Ghaedi, Z. X. Tan, H. H. Ghayeb. (2020). A hybrid ANN-based imperial competitive algorithm methodology for structural damage identification of slab-on-girder bridge using data mining, Appl. Soft Comput. J. 88 (2020) 106013. doi:10.1016/j.asoc.2019.106013.

[17] M. Gordan, Z. Ismail, H. Abdul Razak, K. Ghaedi, Z. Ibrahim, Z. X. Tan, H. H. Ghayeb. (2020). Data mining-based damage identification of a slab-on-girder bridge using inverse analysis, Measurement. 151(2020), 107175. doi:10.1016/j.measurement.2019.107175.

[18] K. Ghaedi, Z. Ibrahim. (2017). Earthquake Prediction, in: T. Zouaghi (Ed.), Earthquakes-Tectonics, Hazard Risk Mitig., InTech, 2017: pp. 205-227. doi:10.5772/65511.

[19] Z. X. Tan, D. P. Thambiratnam, T. H. T. Chan, M. Gordan, H. Abdul Razak. (2019). Damage detection in steel-concrete composite bridge using vibration characteristics and artificial neural network, Struct. Infrastruct. Eng. (2019), 1-15. doi:10.1080/15732479.2019.1696378.

[20] M. Gordan, Z. Ismail, Z. Ibrahim, H. Hashim. (2019). Data Mining Technology for Structural Control Systems: Concept, Development, and Comparison, in: Recent Trends Artif. Neural Networks, IntechOpen Limited, London, 2019. doi:10.5772/intechopen.88651.

[21] M. U. Hanif, Z. Ibrahim, K. Ghaedi, H. Hashim, A. Javanmardi. (2018). Damage assessment of reinforced concrete structures using a model-based nonlinear approach-A comprehensive review, Construction and Building Materials. 192(2018), 846-865.doi:10.1016/j.conbuildmat.2018.10.115.

[22] A. Javanmardi, Z. Ibrahim, K. Ghaedi, N. Bahadur Khan, H. Benisi Ghadim. (2018). Seismic isolation retrofitting solution for an existing steel cable-stayed bridge, PLoS ONE (2018), 13(7).doi.org/10.1371/journal.pone.0200482.

[23] H. H. Ghayeb, H. A. Razak, N. H. R. Sulong, A. N. Hanoon, F. Abutaha, H. A. Ibrahim, M. Gordan, M. F. Alnahhal. (2019). Predicting the Mechanical Properties of Concrete Using Intelligent Techniques to Reduce CO 2 Emissions, Mater. Construcción. 69(2019), 1-20.

[24] S. A. Ravanfar. (2017). Vibration-Based Structural Damage Detection and System Identification Using Wavelet Multiresolution Analysis, Doctor of Philosophy Thesis, University of Malaya, 2017.

[25] M. Gordan, H. A. Razak, Z. Ismail, K. Ghaedi. (2018). Data mining based damage identification using imperialist competitive algorithm and artificial neural network, Lat. Am. J. Solids Struct., 15(2018), 1-14. doi:http://dx.doi.org/10.1590/1679-78254546.

[26] M. Gordan, Z. B. Ismail, H. A. Razak, K. Ghaedi. (2019). Optimization-Based Evolutionary Data Mining Techniques for Structural Health Monitoring, J. Civ. Eng. Constr., 9(2019), 14-23.

[27] W. H. Maes, K. Steppe. (2019). Perspectives for Remote Sensing with Unmanned Aerial Vehicles in Precision Agriculture, Trends Plant Sci., 24(2019), 152-164. doi:10.1016/j.tplants.2018.11.007.

[28] D. Kinaneva, G. Hristov, J. Raychev, P. Zahariev. (2019). Early forest fire detection using drones and artificial intelligence, in: 2nd Int. Conv. Inf. Commun. Technol. Electron. Microelectron. MIPRO 2019-Proc., Croatian Society MIPRO, 2019: pp. 1060-1065. doi:10.23919/MIPRO.2019.8756696.

[29] P. Darby, V. Gopu. (2018). Bridge Inspecting with Unmanned Aerial Vehicles R \& D, 2018.

[30] M. Wazid, A. K. Das, N. Kumar, A. V. Vasilakos, J. J. P. C. Rodrigues. (2019). Design and analysis of secure lightweight remote user authentication and key agreement scheme in internet of drones deployment, IEEE Internet Things J., 6(2019), 3572-3584. doi:10.1109/JIOT.2018.2888821.

[31] K. Ghaedi, Z. Ibrahim, A. Javanmardi, R. Rupakhety. (2018). Experimental study of a new bar damper device for vibration control of structures subjected to earthquake loads. Journal of Earthquake Engineering, (2018), 1-19. doi:10.1080/13632469.2018.1515796.

[32] Y. Dong, Q. Li, A. Dou, X. Wang. (2011). Journal of Asian Earth Sciences Extracting damages caused by the 2008 Ms 8.0 Wenchuan earthquake from SAR remote sensing data, 40(2011), 907-914. doi:10.1016/j.jseaes.2010.07.009.

[33] Y. Pang, B. K. Chen, W. Liu, S. Fung, S. N. Lingamanaik. (2020). Development of a non-contact and non-destructive laser speckle imaging system for remote sensing of anisotropic deformation around fastener holes. NDT E Int. 111(2020), 102219. doi:10.1016/j.ndteint.2020.102219.

[34] M. Talebkhah, A. Sali, M. Marjani, M. Gordan, S. J. Hashim, F. Z. Rokhani. (2020). Edge computing: Architecture, Applications and Future Perspectives, in: IICAIET2020 (IEEE Int. Conf. Artif. Intell. Eng. Technol., Sabah, Malaysia, 2020). 